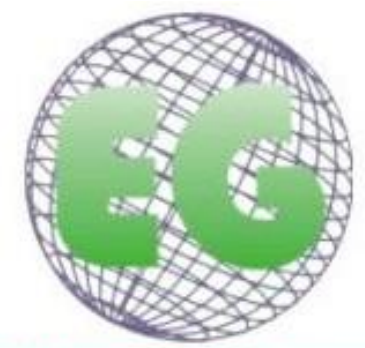

ISSN 1695-6141 $N^{\circ} 48$

\title{
Apoyo social y engagement como antecedentes de la satisfacción laboral en personal de enfermería
}

Social support and engagement as antecedents of job satisfaction in nursing staff

\author{
Alejandro Orgambídez-Ramos ${ }^{1}$ \\ Yolanda Borrego-Alés ${ }^{2}$ \\ ${ }^{1}$ Departamento de Psicologia e Ciências da Educação. Universidade do Algarve. Portugal. \\ ${ }^{2}$ Departamento de Psicología Social, Evolutiva y de la Educación. Universidad de Huelva. España.
}

E-mail: aoramos@ualg.pt

http://dx.doi.org/10.6018/eglobal.16.4.260771

Recibido: 09/06/2016

Aceptado: 30/08/2016

\section{RESUMEN:}

Objetivo: La satisfacción laboral es considerada como uno de los indicadores más importantes tanto de la calidad de los cuidados de salud como de la calidad de vida laboral de los profesionales de la salud. Entre los antecedentes de la satisfacción laboral, se destaca el apoyo social del supervisor y de los colegas y el engagement en el trabajo en el contexto de enfermería. En este sentido, el objetivo de este estudio es comprobar el papel predictor del apoyo social y del engagement en el trabajo sobre la satisfacción laboral en una muestra de enfermeros y enfermeras del sur de Portugal.

Métodos: Estudio transversal a través de cuestionarios. La muestra estuvo compuesta por 215 profesionales de enfermería de hospitales del sur de Portugal (55.56\% de tasa de respuesta).

Resultados: La cohesión social del supervisor y de los compañeros y el engagement en el trabajo se relacionaron de forma positiva y significativa con la satisfacción laboral. Los modelos de regresión lineal múltiple y jerárquica mostraron que los dos tipos de apoyo social y el engagement fueron predicciones significativos de la satisfacción con el trabajo de los participantes.

Conclusión: Intervenciones basadas en la creación de redes informales y formales de apoyo, la formación de los supervisores en competencias de gestión y coaching y una mayor presencia de recursos laborales y personales permitirían aumentar los niveles de satisfacción laboral en los profesionales de enfermería.

Palabras clave: apoyo social; engagement; satisfacción laboral; Portugal

\section{ABSTRACT:}

Objective: Job satisfaction is considered to be one of the most important indicators of quality of health care and quality of work life for health professionals. In the context of nursing, social support and work engagement are regarded as two of the most important predictors of job satisfaction. Consequently, the aim of this study was to examine to what extent social support (supervisor/coworkers) and work engagement predict job satisfaction in a sample of Portugueses nurses. 
Methodology: A cross-sectional study using questionnaires was conducted. A convenience sample of members from Portuguese hospitals was used in this study $(n=215$, return rate $=55.56 \%)$.

Results: Social support from supervisor and from coworkers and work engagement were significantly related to job satisfaction. Regression models showed that job satisfaction was significantly predicted by both types of social support and work engagement.

Conclusion: Interventions based on the creation of informal and formal support networks, training of supervisors in management and coaching skills, and a greater presence of work and personal resources would increase the levels of job satisfaction in nurses.

Keywords: social support; engagement; job satisfaction; Portugal

\section{INTRODUCCIÓN}

Desde la década de los noventa el análisis de las condiciones de trabajo en las organizaciones de salud ha evolucionado desde una perspectiva negativa, centrada en exclusiva en el estrés y el burnout, hacia una perspectiva más positiva y focalizada en mejorar la calidad de vida de las personas ${ }^{(1,2)}$. Este objetivo cobra todavía más relevancia teniendo en cuenta el contexto actual de crisis económica y financiera en los servicios de salud y las características del trabajo realizado por el personal de enfermería: trabajo por turno, trato con usuarios y familias, contacto directo con la enfermedad, el dolor y la muerte, y falta de autonomía para poder tomar decisiones, entre otras ${ }^{(3)}$.

En este contexto, el modelo Demandas-Recursos laborales (DRL) ${ }^{(4,5)}$ aparece como un marco teórico que trata de integrar estas dos perspectivas de estudio: la investigación sobre el estrés y la investigación sobre la motivación y el bienestar. De acuerdo con el modelo DRL ${ }^{(4,5)}$, en cualquier tipo de trabajo se pueden distinguir dos tipos de características: demandas y recursos laborales. Las demandas laborales son características de la organización del trabajo que exigen de la persona un esfuerzo para ser realizadas, y ese esfuerzo lleva asociado un coste físico y/o psicológico (mental o emocional). Ejemplos de demandas laborales serían sobrecarga en el trabajo (p.ej., número excesivo de pacientes por atender), elevada concentración en el trabajo (p.ej., durante la atención sanitaria), demandas físicas (p.ej., movilización de pacientes) o tener que mostrar empatía o emociones positivas cuando se siente lo contrario (p.ej., dar malas noticias a un paciente) ${ }^{(6)}$.

Por el contrario, los recursos laborales serían aquellas características del trabajo necesarias para hacer frente a las demandas. Ejemplos de recursos laborales serían el disponer de feedback sobre el desempeño de las tareas, recursos físicos (p.ej., equipamiento para la movilización de pacientes) o recursos sociales (p.ej., apoyo social de los colegas) (6). Concretamente, los recursos laborales serían aquellas características físicas, psicológicas, sociales u organizacionales que ${ }^{(6)}$ : (a) permiten la consecución de objetivos (p.ej., la autonomía mejora la eficacia), (b) reducen las demandas laborales y sus costes asociados (p.ej., la ayuda de un compañero para atender el exceso de trabajo) y (c) favorecen el crecimiento y el desarrollo personal y profesional (p.ej., formación para adquirir nuevos conocimientos profesionales).

Las demandas laborales elevadas "consumen" a la persona provocando el síndrome de quemarse en el trabajo (burnout) mientras que los recursos laborales se asocian con el engagement de los trabajadores ${ }^{(4,7)}$. Según el modelo DRL, se asume que las demandas y los recursos laborales tienen un impacto directo en los resultados organizacionales a través de los estados psicológicos del burnout y del engagement. 
Relativo al engagement, este se define como un estado mental positivo asociado al trabajo y caracterizado por altos niveles de vigor, dedicación y absorción ${ }^{(8,9)}$. El vigor se refiere a los elevados niveles de energía y resistencia mental cuando se trabaja, asociado al deseo de invertir esfuerzo en el trabajo incluso cuando aparecen dificultades. La dedicación se asocia a la alta implicación laboral, junto con la manifestación de un sentimiento de significado, entusiasmo, orgullo en el trabajo e inspiración. Por último, la absorción aparece cuando la persona está totalmente concentrada en el trabajo mientras el tiempo "pasa volando", con dificultades para desconectar de lo que se está haciendo ${ }^{(6,8,9)}$.

Según Saks ${ }^{(10)}$, el engagement en el trabajo está asociado a importantes actitudes en el trabajo tales como la satisfacción profesional o la poca intención de abandono de la empresa. Dado que los empleados engaged sienten que el trabajo que realizan es importante y muestran un mejor desempeño en sus tareas, estos valoran positivamente la función que realizan y experimentan mayores niveles de satisfacción. Diversos estudios han mostrado relaciones positivas y significativas entre el engagement y la satisfacción laboral con muestras de diversas ocupaciones ${ }^{(11-14)}$, y con muestras de personal de enfermería ${ }^{(15,16)}$.

Entre los recursos laborales considerados por el modelo DRL, el apoyo social es considerado uno de los elementos más importantes para los profesionales de enfermería $^{(17-21)}$. El apoyo social hace referencia al clima social en el lugar de trabajo relativamente a las relaciones con los compañeros y con los supervisores ${ }^{(22)}$. Implica tanto el apoyo socio-emocional como el llamado apoyo social instrumental. Mientras que el primero hace referencia al grado de integración social y emocional entre compañeros de trabajo y supervisores, el segundo se refiere a la colaboración en las tareas del trabajo que se presta entre empleados y supervisores ${ }^{(23)}$.

Diversos estudios han mostrado las relaciones entre las percepciones del personal de enfermería del apoyo social que reciben y la calidad del cuidados ofrecida, la intención de abandonar la organización, el desempeño profesional y la satisfacción laboral ${ }^{(17-21)}$. Igualmente se ha observado que elevados niveles de apoyo social, tanto del supervisor como de los colegas, amortiguan los efectos negativos del estrés y el burnout sobre la satisfacción laboral $(18,21)$. El intercambio de experiencias con los compañeros y supervisores, el feedback recibido por los supervisores y las buenas relaciones interpersonales en el contexto de trabajo aumentan los sentimientos y sensaciones de bienestar en el trabajo, aumentando los niveles de satisfacción laboral experimentados por el personal de enfermería.

El estudio de la satisfacción laboral cobra una mayor relevancia en el contexto de los servicios de salud y especialmente entre los profesionales de enfermería (24). La satisfacción laboral puede ser considerada tanto como un indicador indirecto de la calidad, tanto asistencial como de los servicios prestados, de las instituciones sanitarias como de la calidad de vida laboral de sus miembros ${ }^{(24,25)}$. No obstante, la investigación sobre las relaciones entre engagement, apoyo social y satisfacción laboral es todavía escasa y prácticamente no se ha llevado a cabo en el contexto de salud de Portugal. En ese sentido, el objetivo de este estudio es comprobar el papel predictor del apoyo social y del engagement en el trabajo sobre la satisfacción laboral en una muestra de enfermeros y enfermeras del sur de Portugal. 


\section{MATERIALES Y MÉTODOS}

\section{Diseño y muestra}

Se llevó a cabo un estudio transversal, descriptivo y correlacional mediante el uso de cuestionarios ${ }^{(26)}$. 450 profesionales de enfermería de varios hospitales públicos del sur de Portugal fueron encuestados, obteniéndose una muestra una final de 215 participantes $(55.56 \%$ de tasa de respuesta). El $77,21 \%$ de la muestra son mujeres, siendo la edad media de la muestra de 34,89 años $(D P=9,37)$. En relación al estado civil, $46,51 \%$ de los participantes expresó estar solteros y el $34,8 \%$ casados. Cerca del $71 \%(70,75 \%)$ de la muestra posee contrato indefinido y los años de experiencia profesional en enfermería ronda los 10 años $(M=9,40, D P=8,10)$.

\section{Instrumentos}

Apoyo social. Para evaluar el apoyo social se utilizó la adaptación al portugués de la dimensión de apoyo en el trabajo del cuestionario Job Content Questionnaire (JCQ) $(22,27)$. Los 11 ítems de la escala se distribuyen en dos subescalas: apoyo del supervisor (5 ítems) y apoyo de los compañeros (6 ítems). Las respuestas fueron dadas en una escala Likert de 1: Totalmente en desacuerdo a 4: Totalmente de acuerdo. Mayores puntuaciones indican elevados niveles de apoyo social tanto del supervisor como de los compañeros. Los coeficientes de fiabilidad (alpha de Cronbach) de las dimensiones apoyo del supervisor y apoyo de los compañeros fueron de 0,93 y 0,86 , respectivamente.

Engagement en el trabajo. Para medir el engagement en el trabajo fue utilizada la versión portuguesa del Utrecht Work Engagement Scale (UWES) ${ }^{(8)}$. Esta escala consta de 9 ítems distribuidos en tres dimensiones: vigor ( 3 ítems), dedicación (3 ítems) y absorción (3 ítems). Los participantes respondían a los 9 ítems utilizando una escala Likert desde 0: Nunca a 6: Todos los días. Elevadas puntuaciones expresan elevados niveles de engagement en el trabajo por parte de los participantes. El coeficiente alpha de Cronbach obtenido en este estudio fue de 0,92.

Satisfacción en el trabajo. Para evaluar la satisfacción en el trabajo se utilizó la escala de Lima, Vala y Monteiro ${ }^{(28)}$. Esta escala está compuesta por 8 ítems que miden la satisfacción con diversos aspectos de trabajo. Los participantes responden a cada enunciado utilizando una escala Likert desde 1: Totalmente en desacuerdo a 7: Totalmente de acuerdo. Mayores puntuaciones expresan elevados niveles de satisfacción con el trabajo. El coeficiente de consistencia interna (alpha de Cronbach) registrado en este estudio fue de 0,87 .

Recogida de datos. La batería de cuestionario fue aplicada entre febrero y abril de 2015. Tras el pedido de autorización a los hospitales, todos los comités éticos aprobaron el estudio. Los cuestionarios fueron aplicados individualmente 0 en pequeños grupos al principio del turno laboral, dando todo el tiempo necesario a los participantes. La participación fue totalmente voluntaria y en todo momento se garantizó la confidencialidad y anonimato de todos los datos.

Análisis de datos. El análisis de datos se realizó utilizando el paquete estadístico STATA v13. Se calcularon los estadísticos descriptivos de las variables de la investigación (media, desviación típica, asimetría, curtosis), las correlaciones entre ellas y los coeficientes de consistencia interna (alpha de Cronbach). Modelos de 
regresión lineal múltiple y jerárquica fueron llevados a cabo para comprobar el papel predictor del apoyo social y el engagement sobre la satisfacción laboral.

\section{RESULTADOS}

\section{Análisis preliminares}

Antes de verificar los objetivos propuestos, se realizaron varias pruebas para evaluar el efecto de la varianza del método común (Common Method Variance, CMV). Dado que todos los datos fueron recogidos mediante medidas de autoinforme y en el mismo intervalo de tiempo, la varianza común asociada al método puede sobreestimar o infravalorar las relaciones entre las variables ${ }^{(29)}$. El test one-factor de Harman fue realizado para comprobar el posible efecto de la varianza común. Todos los ítems de la escala de apoyo social, engagement y satisfacción laboral fueron sometidos a un análisis factorial exploratorio mediante el método de componentes principales, con rotación varias y forzando la extracción de un solo factor. Si hubiera un problema de varianza del método común, el factor extraído debería dar cuenta de más del $50 \%$ de la varianza. Los resultados del análisis factorial mostraron un factor que daba cuenta del $44,33 \%$. Aunque no puede descartarse totalmente el efecto de la varianza común, no parece afectar significativamente a las relaciones entre las variables estudiadas ${ }^{(29)}$.

\section{Descriptivos y correlaciones}

En la Tabla 1 se muestran las medias, desviaciones típicas, asimetría, curtosis y correlaciones de las variables del estudio, así como la fiabilidad de las escalas. El apoyo del supervisor $(\mathrm{M}=2,96, \mathrm{DP}=0,88)$ y el apoyo de los compañeros $(\mathrm{M}=3,07$, $\mathrm{DP}=0,62$ ) se situaron por encima de la media de la escala de respuesta. En relación al engagement, los profesionales de enfermería mostraron elevados bastante elevados $(M=4,61, D P=1,06)$ mientras que la satisfacción con el trabajo se situó ligeramente por encima de la media de la escala de respuesta $(M=4,39, D P=1,14)$.

Tabla 1: Estadísticos descriptivos y correlaciones de todas las variables del estudio

\begin{tabular}{lllll}
\hline Variable & 1 & 2 & 3 & 4 \\
\hline 1. Apoyo del supervisor & $(0,92)$ & 0,45 & 0,30 & 0,67 \\
2. Apoyo de los compañeros & & $(0,93)$ & 0,41 & 0,67 \\
3. Engagement en el trabajo & & & $(0,86)$ & 0,62 \\
4. Satisfacción laboral & & & & $(0,87)$ \\
\hline Media & 4,61 & 2,96 & 3,07 & 4,39 \\
Desviación Típica & 1,06 & 0,88 & 0,62 & 1,14 \\
Asimetría & $-1,25$ & $-0,65$ & $-0,40$ & $-0,06$ \\
Curtosis & 1,59 & $-0,53$ & 0,28 & $-0,31$ \\
\hline
\end{tabular}

Nota: El coeficiente de fiabilidad se encuentra en la diagonal entre paréntesis

Todos los coeficientes son estadísticamente significativos $(p<0,01)$

Como fue esperado, los análisis de correlación mostraron que tanto el apoyo social como el engagement en el trabajo se relacionaron de forma significativa $(p<0,01)$ con la satisfacción en el trabajo: 0,67 con el apoyo del supervisor, 0,67 con apoyo de los compañeros y 0,62 con el engagement en el trabajo. Mayores puntuaciones en apoyo social (supervisor y colegas) y en engagement en el trabajo se relacionaron con mayores puntuaciones en satisfacción en el trabajo. 


\section{Modelos de regresión lineal múltiple}

Para comprobar el objetivo del estudio, se llevaron a cabo varios modelos de regresión lineal múltiple y jerárquica. Estos modelos se utilizaron para verificar el papel predictor del apoyo social (supervisor y compañeros) y el engagement en el trabajo sobre los niveles de satisfacción laboral de los participantes. Antes del análisis, se comprobó que los supuestos de normalidad y homoscedasticidad no se violaron.

En primer lugar, considerando el modelo de Demandas-Recursos laborales, en el primer paso (M1) se incluyeron el apoyo del supervisor y el apoyo de los compañeros como predictores de la satisfacción laboral en el modelo jerárquico de regresión. El total de la varianza explicada por el modelo fue del 58,62\%, $F(2,197)=139,55$, $\mathrm{p}<0,01$. Los dos tipos de apoyo organizacional se mostraron como predicciones significativos $(p<0,01)$, con un valor Beta de 0,50 para apoyo del supervisor y un valor Beta de 0,39 para apoyo de los compañeros.

Tabla 2: Modelos de regresión lineal múltiple y jerárquica para satisfacción laboral

\begin{tabular}{lllll}
\hline & Beta & $S E$ & $t$ & VIF \\
\hline Modelo 1 (M1) & & & & \\
- Apoyo del supervisor & 0,50 & 0,05 & 10,05 & 1,22 \\
- Apoyo de los compañeros & 0,39 & 0,05 & 7,87 & 1,22 \\
\hline Modelo 2 (M2) & & & & \\
- Apoyo del supervisor & 0,35 & 0,04 & 7,98 & 1,39 \\
- Apoyo de los compañeros & 0,33 & 0,04 & 7,96 & 1,25 \\
- Engagement en el trabajo & 0,41 & 0,04 & 10,05 & 1,27 \\
\hline
\end{tabular}

Nota: Todos los coeficientes son estadísticamente significativos $(p<0,01)$

En segundo y último lugar, se añadió al modelo (M2) la variable engagement en el trabajo como variable independiente. La varianza total explicada en el modelo M2 fue del $72,39 \%, F(3,196)=173,90, p<0,01$. El engagement en el trabajo explicó un 14,10 de varianza adicional, cambio en $\mathrm{R} 2=0,14, \mathrm{~F}(1,196)=100,97, \mathrm{p}<0,01$, tras controlar el efecto del apoyo del supervisor y del apoyo de los compañeros. En este último modelo todas las variables independientes fueron predictores significativos $(p<0,01)$, con un valor Beta de 0,35 para apoyo del supervisor, un valor Beta de 0,33 para apoyo de los compañeros, y un valor Beta de 0,41 para engagement en el trabajo.

\section{DISCUSIÓN}

La satisfacción laboral es uno de los elementos más importantes de la calidad de vida laboral de los profesionales de enfermería, por su impacto tanto en la calidad de los cuidados ofrecidos y en la relación profesional-paciente, como en la salud y bienestar de las personas ${ }^{(24)}$. Entre los antecedentes de la satisfacción en enfermeros y enfermeras, cabe destacar el engagement en el trabajo y el apoyo social de supervisores y compañeros ${ }^{(15-18,20,21)}$. En este sentido, el objetivo de estudio fue comprobar el papel predictor del apoyo social y del engagement en el trabajo sobre la satisfacción laboral en una muestra de enfermeros y enfermeras del sur de Portugal. Los resultados de la investigación mostraron la relación positiva entre engagement en el trabajo y apoyo social, y la satisfacción laboral experimentada. Además, tanto el engagement en el trabajo como el apoyo de supervisores y de compañeros se mostraron como predictores significativos de la satisfacción en el trabajo conforme al 
Los resultados mostraron la relación positiva y significativa entre el engagement en el trabajo y la satisfacción laboral, en línea con los estudios realizados por Spence Laschinger ${ }^{(15)}$ y Van Bogaert et al. ${ }^{(16)}$. Cuando los profesionales de enfermería se sienten engaged en el trabajo, muestran mayores niveles de desempeño en el trabajo y valoran de un modo mucho más positivo las tareas que realizan. En consecuencia, valoran positivamente su contexto de trabajo manifestando mayores niveles de satisfacción en el trabajo ${ }^{(11-14,16)}$.

En la misma línea, los resultados también recogieron la relación positiva entre el apoyo social, tanto del supervisor como de los compañeros, y la satisfacción en el trabajo. La comunicación con el supervisor y los compañeros, la posibilidad de recibir apoyo y retroalimentación del supervisor, el recibir consejos útiles para la realización de las tareas por parte de los colegas y el soporte para afrontar situaciones "estresantes" permite la autorregulación de los pensamientos y sentimientos en el contexto de trabajo ${ }^{(17,18,21)}$. Todo ello favorece una evaluación mucho más positiva del contexto de trabajo asociándolo a sentimientos y emociones positivas, incrementado los niveles de satisfacción laboral. En este sentido, los resultados de la investigación son consistentes con la literatura científica sobre las relaciones entre apoyo social y satisfacción laboral en personal de enfermería ${ }^{(17-21)}$.

Los modelos de regresión indicaron que el apoyo del supervisor, el apoyo de los compañeros y el engagement en el trabajo fueron los predictores positivos y significativos de la satisfacción, siendo el engagement el predictor más potente. Estos resultados están en línea con otras investigaciones realizadas con muestras de enfermeros y enfermeras ${ }^{(15-18)}$. Los valores Beta obtenidos son semejantes a los obtenidos por AbuAIRub y colaboradores ${ }^{(17,18)}$ y por Spence Laschinger ${ }^{(15)}$. A través del apoyo social los profesionales de enfermería reciben orientaciones y feedback sobre el desempeño y las tareas que realizan. Además, el apoyo social, tanto de supervisores como de colegas, ayuda a la expresión de las emociones negativas y minimiza la sensación de soledad que pueden sentir estos profesionales ${ }^{(19,21)}$. Todo ello favorece la experiencia positiva de satisfacción laboral. Por su parte, los estados psicológicos asociados al engagement aumentan la capacidad de afrontamiento de las personas en el trabajo, haciéndoles percibir positivamente su ambiente de trabajo y aumentando la satisfacción laboral $(4,5,15,16)$.

Este estudio tiene una serie de limitaciones que deben tenerse en cuenta. En primer lugar, el diseño transversal y correlacional no permite establecer relaciones de causalidad entre las variables. No obstante, el modelo de regresión utilizado plantea la existencia de variables independientes y dependientes ya que el modelo DRL permite establecer estas relaciones entre las variables del estudio ${ }^{(4,5)}$. Un segundo elemento a tener es la varianza asociado al método común. Si bien no parece afectar al análisis de datos, no puede descartarse totalmente su influencia ${ }^{(29)}$. Por otro lado, el diseño de encuestas es especialmente sensible a determinados sesgos, como el sesgo de deseabilidad en las respuestas dadas o la tendencia en las respuestas de aquellas personas que voluntariamente colaboraron en el estudio ${ }^{(30)}$.

Finalmente, el apoyo social (de supervisores y de colegas) y el engagement en el trabajo aparecen como elementos que permiten el diseño de intervenciones para aumentar la satisfacción laboral en profesionales de enfermería. Entre las estrategias de mejora del apoyo social, cabe destacar la creación de redes formales e informales 
dentro de los servicios de salud y la formación de los supervisores en estilos participativos y democráticos de liderazgo. Dado el papel fundamental de los supervisores en el apoyo social, sería necesaria una formación continuada que posibilite el desarrollo de competencias de gestión, asesoramiento y supervisión.

Otra línea de actuación se centraría en el aumento de los niveles de engagement en el lugar de trabajo aumentando los recursos laborales y personales en el trabajo. Recursos organizacionales como la autonomía o la formación afectan de forma positiva a las tres dimensiones del engagement. Igualmente, el acceso a recursos (materiales, tiempo, informaciones técnicas) y la oportunidad de aplicar y desarrollar nuevas competencias favorece la aparición de contextos laborales que potencien el engagement de los profesionales de enfermería.

\section{REFERENCIAS}

1. García-Rodríguez JJ, Labajos MT, Fernández-Luque F. Los estudiantes de Grado de Enfermería y su compromiso con los estudios. Enfermería Global. 2015;14:169-176.

2. Snyder CR, López J. The Oxford handbook of positive psychology. New York: Oxford University Press; 2009

3. Gil-Monte PR, Carretero-Dios H, Roldán MD. Algunos procesos psicosociales sobre el síndrome de quemarse por el trabajo (burnout) en profesionales de enfermería. Ansiedad y Estrés. 2005;11:281-291.

4. Bakker AB, Demerouti E. Job demands-resources model. Journal of Work and Organizational Psychology. 2013;29:107-115.

5. Demerouti E, Bakker AB. The job demands-resources model: Challenges for future research. SA Journal of Industrial Psychology. 2011;37:1-9.

6. Salanova M, Schaufeli WB. El engagement en el trabajo. Cuando el trabajo se convierte en pasión. Madrid: Alianza Editorial; 2009

7. Bakker AB, Demerouti E. Toward a model of work engagement. Career Development International. 2008;13:209-223.

8. Schaufeli WB, Salanova M, González-Romá V, Bakker AB. The measurement of engagement and burnout: A two sample confirmatory factor analytic approach. Journal of Happiness Studies. 2002;3:71-91.

9. Schaufeli WB, Bakker AB. Job demands, job resources, and their relationships with burnout and engagement: A multi-sample study. Journal of Organizational Behavior. 2004;25:293-315.

10. Saks AM. Antecedents and consequences of employee engagement. Journal of Managerial Psychology. 2006;21:600-619.

11. Alarcon GM, Lyons JB. The relationship of engagement and job satisfaction in working samples. The Journal of Psychology. 2011;145:463-480.

12. Durán MA, Extremera N, Montalbán FM, Rey L. Engagement y burnout en el ámbito docente: Análisis de sus relaciones con la satisfacción laboral y vital en una muestra de profesores. Revista de Psicología del Trabajo y de las Organizaciones. 2005;21:145-158.

13. Moura D, Orgambídez-Ramos A, Gonçalves G. Role stress and work engagement as antecedents of job satisfaction: Results from Portugal. Europe's Journal of Psychology. 2014;10:291-300.

14. Orgambídez-Ramos A, Borrego-Alés Y, Mendoza-Sierra MI. Role stress and work engagement as antecedents of job satisfaction in Spanish workers. Journal of Industrial Engineering and Management. 2014;7:360-372.

15. Spence Laschinger HK. Job and career satisfation and turnover intentions of 
newly graduated nurses. Journal of Nursing Management. 2012;20:472-484.

16. Van Bogaert $P$, Wouters $K$, Willems R, Mondelaers M, Clarke S. Work engagement supports nurse workforce stability and quality of care: Nursing team-level analysis in psychiatric hospitals. Journal of Psychiatric and Mental Health Nursing. 2013;20:679-686.

17. AbuAIRub RF, Omari FH, Al-Zaru IM. Support, satisfaction and retention among Jordanian nurses in private and public hospitals. International Nursing Review. 2009;56:326-332.

18. AbuAIRub RF, Omari FH, Rub A. The moderating effect of social support on the stress-satisfaction relationship among Jordanian hospital nurses. Journal of Nursing Management. 2009;17:870-879.

19. Bartram T, Joiner TA, Stanton P. Factors affecting the job stress and job satisfaction of australian nurses: Implications for recruitment and retention. Contemporary Nurse. 2004;17:293-304.

20. Cortese C, Colombo L, Ghislieri C. Determinants of nurses' job satisfaction: The role of work-family conflict, job demand, emotional charge and social support. Journal of Nursing Management. 2010;18:35-43.

21. Hamaideh SH. Burnout, social support, and job satisfaction among Jordanian mental health nurses. Issues in Mental Health Nursing. 2011;32:234-249.

22. Karasek R, Theorell T. Healthy work. Stress, productivity, and the reconstruction of working life. New York: BasicBooks; 1990

23. Martín-Arribas MC. Estrés relacionado con el trabajo (modelo de demandacontro-apoyo social) y alteraciones en la salud: Una revisión de la evidencia existente. Enfermería Intensiva. 2007;18:168-181.

24. Carrilo-García C, Ríos-Rísquez MI, Fernández-Cánovas ML, Celdrán-Gil F, Vivo-Molina C, Martínez-Roche ME. La satisfacción de la vida laboral de los enfermeros de las unidades móviles de emergencias de la Región de Murcia. Enfermería Global. 2015;14:266-275.

25. Peiró JM, Silla I, Sanz T, Rodríguez J, García J. Satisfacción laboral de los profesionales de Atención Primaria. Psiquis: Revista de Psiquiatría, Psicología Médica y Psicosomática. 2004;25:5-16.

26. Montero I, León OG. A guide for naming research studies in Psychology. International Journal of Clinical and Health Psychology. 2007;7:847-862.

27. Escribá-Agüir V, Más R, Flores E. Validación del Job Content Questionnaire en personal de enfermería hospitalario. Gaceta Sanitaria. 2001;15:142-149.

28. Lima M, Vala J, Monteiro MB. Culturas organizacionais. In: Vala MB, Monteiro MB, Caetano A, editors. Psicologia social e das organizações - Estudos em empresas portuguesas. Lisboa: Celta Editora; 1994.

29. Podsakoff PM, MacKenzie S, Podsakoff NP. Source of method bias in social science research and recommendations on how to control it. Annual Review of Psychology. 2012;63:539-569.

30. Navas JM. Métodos, diseños y técnicas de investigación psicológica. Madrid: UNED; 2002

ISSN 1695-6141

๑) COPYRIGHT Servicio de Publicaciones - Universidad de Murcia 